\title{
Influence of antisite defects and stacking faults on the magnetocrystalline anisotropy of FePt
}

\author{
M. Wolloch, ${ }^{1,2, *}$ D. Suess, ${ }^{3,4}$ and P. Mohn ${ }^{2}$ \\ ${ }^{1}$ Department of Physics, Informatics and Mathematics, University of Modena and Reggio Emilia, Via Campi, 213/A 41125 Modena, Italy \\ ${ }^{2}$ Institute of Applied Physics, Vienna University of Technology, Wiedner Hauptstr. 8-10/134, 1040 Vienna, Austria \\ ${ }^{3}$ University of Vienna, Faculty of Physics, Währingerstrasse 17, 1090 Vienna, Austria \\ ${ }^{4}$ Institute of Solid State Physics, Vienna University of Technology, Wiedner Hauptstr. 8-10/134, 1040 Vienna, Austria
}

(Received 30 March 2017; revised manuscript received 7 July 2017; published 7 September 2017)

\begin{abstract}
We present density functional theory (DFT) calculations of the magnetic anisotropy energy (MAE) of FePt, which is of great interest for magnetic recording applications. Our data, and the majority of previously calculated results for perfectly ordered crystals, predict a MAE of $\sim 3.0 \mathrm{meV}$ per formula unit, which is significantly larger than experimentally measured values. Analyzing the effects of disorder by introducing stacking faults (SFs) and antisite defects (ASDs) in varying concentrations we are able to reconcile calculations with experimental data and show that even a low concentration of ASDs are able to reduce the MAE of FePt considerably. Investigating the effect of exact exchange and electron correlation within the adiabatic-connection dissipation fluctuation theorem in the random phase approximation (ACDFT-RPA) reveals a significantly smaller influence on the MAE. Thus the effect of disorder, and more specifically ASDs, is the crucial factor in explaining the deviation of common DFT calculations of FePt to experimental measurements.
\end{abstract}

DOI: 10.1103/PhysRevB.96.104408

\section{INTRODUCTION}

Storage density of hard disk drives (HDDs) has increased over 8 orders of magnitude since their first introduction in the 1950 s, peaking in more than $100 \%$ increase per year in the late 1990s and reaching $100 \mathrm{~Gb} / \mathrm{in}^{2}$ in 2002 and, after a period of slower growth, finally $500 \mathrm{~Gb} / \mathrm{in}^{2} 2010$ [1]. This tremendous achievement was mainly realized through minimization of the read-write head, thinner recording media and reduced grain size. However, to keep storage density growing, grain sizes need to be further reduced which then can be effected by the superparamagnetic limit. Here the magnetic energy stored in a single grain, the product of grain volume $V$ and magnetic anisotropy constant $K_{u}$, approaches the size of thermal energy $k_{\mathrm{B}} T$. The thermal stability requirements have thus shifted the focus to materials with very high $K_{u}$, especially FePt alloys [2]. While the anisotropy constant of ordered FePt is large enough to allow storage densities of up to $4 \mathrm{~Tb} / \mathrm{in}^{2}$ [3], a further problem arises with the limited write fields employed by conventional read-write heads [4]. Two promising solutions to this problem have been proposed, giving a thermal write assist using a laserpuls concentrated by near field laser optics [5], or exchange spring coupled multilayer media [6,7], which reduce the switching field while maintaining good thermal stability. A combination of both approaches is especially promising, for example using $\mathrm{FePt}$ and $\mathrm{FeRh}$ in a multilayer configuration, so only a small thermal assist to trigger the first-order magnetic phase transition of FeRh (see Ref. [8] and references therein) is needed to write on extremely hard magnetic FePt alloys [9]. To date, the highest demonstrated recording density of

\footnotetext{
*michael.wolloch@unimore.it
}

Published by the American Physical Society under the terms of the Creative Commons Attribution 4.0 International license. Further distribution of this work must maintain attribution to the author(s) and the published article's title, journal citation, and DOI.
$1.402 \mathrm{~Tb} / \mathrm{in}^{2}$ was reported in 2015 by using FePt with heat assisted magnetic recording (HAMR) [10].

Stoichiometric FePt exists both in the disordered fcc $A 1$ phase, and the ordered tetragonal $L 1_{0}$ phase, where $\mathrm{Fe}$ and Pt layers are alternating along the $c$ direction. The extremely large magnetic anisotropy energy (MAE) is only found for the ordered phase, which is stable below $\sim 1300^{\circ} \mathrm{C}$ [1]. For magnetic recording, thin films of the material are mainly fabricated by co-sputtering from elemental or alloyed targets [11-15], and by electron beam evaporation [16,17], but molecular beam epitaxial deposition and other methods are also feasible [1]. The substrate is mainly $\mathrm{MgO}(001)$ and deposition temperature as well as sputtering gas pressure have a large effect on the degree of ordering [12]. Growing extremely highly ordered FePt films with small grains is a challenging endeavor [1], but extensive research is performed in order to improve the growth of grains with the easy axis perpendicular to the film plane using special buffer and seed layers [18]. This helps with the reduction of the in-plane components of the magnetization, which are a serious noise sources in HAMR.

Computationally, it is of course much easier to investigate fully ordered $\mathrm{FePt}$ using periodic boundary conditions then simulating disordered structures. However, even though $\mathrm{FePt}$ has an extremely large MAE, it is still only in the meV range per formula unit (f.u.) and calculated by subtracting comparatively large numbers from each other. Additionally, the MAE is a Fermi surface effect and thus very sensitive to the $k$-point sampling of the Brillouin zone. These effects make accurate calculation challenging and one should not be surprised to find large variations in the results of $a b$ initio calculations in the literature published in the last decades. In Fig. 1, we sort 29 previously calculated values for the MAE of FePt, published in 19 different papers [19-37], in 0.25-meV wide bins and fit the data with a Gaussian distribution. All results have been calculated $a b$ initio with density functional theory (DFT), but involve multiple codes, methods, lattice parameters, and exchange-correlation potentials. Nearly half 


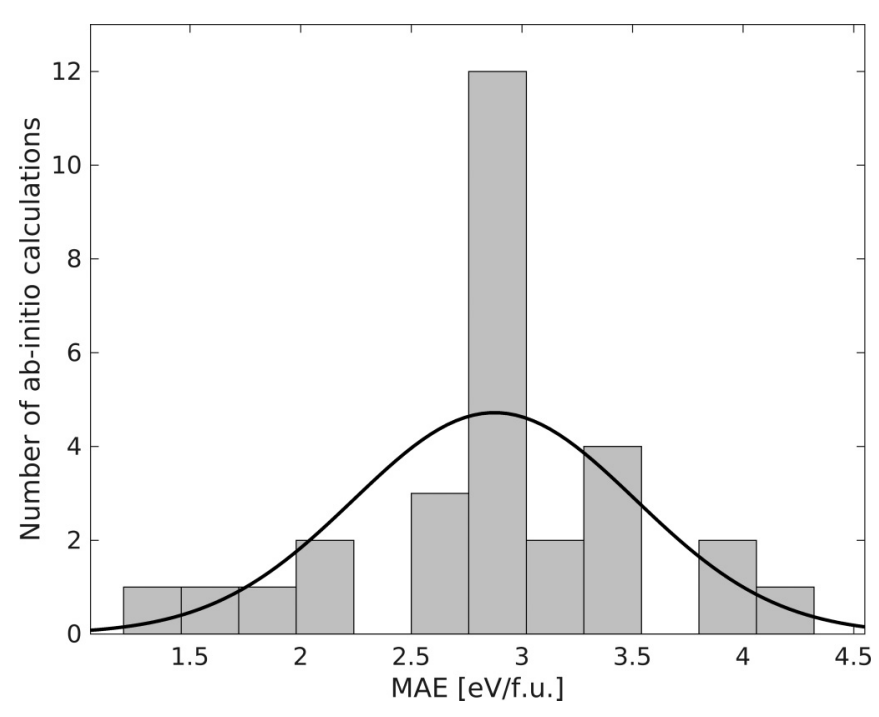

FIG. 1. Histogram of 29 previously calculated MAE values of FePt with different DFT codes, computational parameters and exchange and correlation approximations. The black curve is a Gaussian fitted to the data resulting in $\mu=2.88 \mathrm{meV} /$ f.u. and $\sigma=0.64 \mathrm{meV} /$ f.u. The data are collected from Refs. [19-37].

of the results fall into the bin between 2.75 and $3.00 \mathrm{meV}$, but the rest of the data are quite scattered, ranging from 1.30 to $4.3 \mathrm{meV} /$ f.u. This leads to an mean of $\mu=2.88 \mathrm{meV} /$ f.u. but quite a large standard deviation of $\sigma=0.64 \mathrm{meV} /$ f.u.

These values are often compared with the bulk experiments of Ivanov et al. [38] from 1973, who used the ballistic throw method and also a vibration magnetometer to measure the magnetic properties of annealed FePt powders, reporting an anisotropy constant of $K_{1}=7.0 \mathrm{MJ} / \mathrm{m}^{3}$, corresponding to $\sim 1.2 \mathrm{meV} /$ f.u.. Looking at the published computational data, only the results by Shick et al. [35] and Staunton et al. [37] are close to this value, at 1.30 and $1.70 \mathrm{meV} /$ f.u., respectively. Ivanov et al. argue that their sample is fully ordered, because it exhibits especially high magnetic anisotropy, but take no further measures to actually quantify the degree of order. From more recent experiments on thin films and powders, however (see Sec. III B 1), we know that full order is not necessary to measure anisotropies of $7.0 \mathrm{MJ} / \mathrm{m}^{3}$ or higher in $\mathrm{FePt}$, leading us to believe that the sample of Ivanov et al. was indeed highly, but not fully ordered. ${ }^{1}$

In a very recent paper, Khan et al. published calculations for the MAE of fully ordered FePt [28]. They employ both full-potential linear augmented plane wave (FLAPW) and full-potential Korringa-Kohn-Rostoker (KKR) Green function methods to calculate the MAE within the local density approximation (LDA), with both methods being in good agreement with each other. We consider those calculation among the most accurate ones published to date and want to compare our results to their paper especially, since the authors

\footnotetext{
${ }^{1}$ Reference [38] provide their $K_{1}$ values for room temperature and above. If we extrapolate the data linearly to $0 \mathrm{~K}$, we arrive at $\sim 10 \mathrm{MJ} / \mathrm{m}^{3}$, corresponding to $\sim 1.7 \mathrm{meV} /$ f.u.. This is closer, but still substantially different, from the mean value of the DFT data.
}

took exceptional care to report convergence data on all relevant computational parameters. However, since their calculated MAE of $\sim 3.0 \mathrm{meV}$ is still about twice as large as the reported experimental bulk value of Ref. [38], Khan et al. conclude that many body effects beyond the LDA are playing a decisive role for the MAE of FePt. In the present paper, we will argue that the results of Ref. [28] and the majority of other computations (see Fig. 1) are indeed correct for ideally ordered FePt, but that experiments always measure somewhat disordered structures and thus defects must be explicitly considered to reconcile calculations with experiment.

\section{METHODOLOGICAL DETAILS}

We have performed spin polarized DFT computations employing the Vienna $a b$ initio simulation package VASP [39-42] version 5.4.1, using the projector augmented-wave (PAW) method $[43,44]$. The plane-wave energy cutoff was chosen to be $900 \mathrm{eV}$, which is more than $230 \%$ (300\%) higher than the recommended value for the Fe (Pt) PAW potentials (set of 2003) which treat the $3 s, 3 p, 3 d$, and $4 s(5 s, 5 p, 5 d$, and $6 s$ ) electrons (32 per FePt pair) as valence. We sample the Brillouine zone with generalized Monkhorst-Pack grids as described by Wisesa et al., finding a significantly quicker convergence with their server generated grids than for those generated by the VASP routines [45]. Unless otherwise noted their parameter $r_{\min }$, which describes the distance between lattice points on the real-space superlattice and increases $k$-mesh density if increased, was set to $65 \AA$. The chosen energy cutoff might seem large, but only with this cutoff we can achieve a total energy convergence of less than $0.1 \mathrm{meV}$, and thus properly quantify the MCA. Unless otherwise noted, the exchange and correlation energy has been calculated within the generalized gradient approximation (GGA), using the functional parametrized by Perdew, Burke, and Ernzerhof (PBE) [46]. To ensure accurate forces during relaxations, we use an additional superfine fast Fourier transform (FFT) grid for the evaluation of the augmentation charges and a smearing of $\leqslant 0.1 \mathrm{eV}$ according to Methfessel and Paxton [47] (first order). For total energy calculations, the tetrahedron method with Blöchl corrections has been used [48]. In all total energy GGA calculations we explicitly account for nonspherical contributions of the gradient corrections inside the PAW spheres. Electronic relaxations are converged to $10^{-5} \mathrm{meV}$, while forces in ionic relaxations where converged to $\leqslant$ $1 \mathrm{meV} / \AA$. For all calculations of the MAE, we turned all symmetry options off explicitly and subtracted the total energy values of hard and easy axis orientation of the magnetic moments. The easy axis is clearly the [001] direction with a hard plane orthogonal to it in which the energy difference between orientations is in the $\mu \mathrm{eV}$ range. We chose the [110] direction of the $L 1_{0}$ unit cell as the hard axis (corresponding to the [001] direction if distorted fcc unit cell is used).

\section{RESULTS AND DISCUSSION}

\section{A. Pristine FePt}

The high MAE of FePt in the $\mathrm{L} 1_{0}$ phase is mainly due to the large spin-orbit coupling in the Pt atoms. They show 
TABLE I. Local spin moments $\left(m^{\text {loc }}\right)$ and orbital magnetic moments $\left(m^{\text {orb }}\right)$ for FePt. $\|$ and $\perp$ mark orientation of the spin moments parallel and normal to the $c$ axis, respectively.

\begin{tabular}{lcccccc}
\hline \hline & \multicolumn{2}{c}{$m^{\text {loc }}\left(\mu_{\mathrm{B}}\right)$} & \multicolumn{4}{c}{$m^{\text {orb }}\left(\mu_{\mathrm{B}}\right)$} \\
& $\mathrm{Fe}$ & $\mathrm{Pt}$ & $\mathrm{Fe}_{\|}$ & $\mathrm{Pt}_{\|}$ & $\mathrm{Fe}_{\perp}$ & $\mathrm{Pt}_{\perp}$ \\
\hline PBE & 2.83 & 0.39 & 0.056 & 0.044 & 0.052 & 0.057 \\
LDA & 2.69 & 0.37 & 0.058 & 0.043 & 0.052 & 0.055 \\
LDA $+U$ & 2.83 & 0.36 & 0.056 & 0.043 & 0.054 & 0.055 \\
\hline \hline
\end{tabular}

magnetic moments induced by the Fe $3 d$ orbitals, and the $d$ orbital of both species hybridize with each other. Detailed discussions about the origin and nature of the large MAE can be found in the literature $[23,28,29,36]$ and will not be discussed here further.

In contrast to Ref. [28], we use the PBE functional, which belongs to the class of GGAs, instead of the LDA. This choice was made due to the better equilibrium volume obtained by PBE of $28.02 ; \AA^{3}$, which at $+2 \%$, is much closer to the experimental value of $27.5 \AA^{3}$ [31] than LDA at $24.55 \AA^{3}(-11 \%)$. Since we need to relax our structure once we introduce defects, getting better volumes and forces is even more important. Additionally, at the smaller equilibrium volume given by the LDA, the local magnetic moments of the Fe and Pt atoms would be reduced by about $15 \%$ and $10 \%$, respectively.

Relaxing $L 1_{0}$ FePt with PBE yields lattice parameters $a=$ $2.7287 \AA$ and $c=3.7629 \AA$. This leads to a $c / a$ ratio of 1.38 , about $1.5 \%$ larger than the experimental value of 1.36 [31]. While this difference will influence the MAE somewhat, the effect of the $c / a$ ratio is considered to be small compared to disorder in the sample [32].

For PBE we calculate an MAE of $2.74 \mathrm{meV} /$ f.u., corresponding to an anisotropy constant $K_{u}$ of $15.7 \mathrm{MJ} / \mathrm{m}^{3}$. Increasing the total number of $k$ points in the Brillouin zone by $\sim 55 \%$ from 7317 to 11340 (via adjusting $r_{\text {min }}$ to $75 \AA$ ) does not change this value. This result compares well to the mean value of previously published results of Fig. 1, and is in excellent agreement with the calculations of Ref. [28], which reports a value of $2.73 \mathrm{meV} / \mathrm{f}$.u. for PBE. The angular dependence of the anisotropy Energy $E$ can be fitted to $E(\theta)-E(0)=K_{1} \sin ^{2}(\theta)+K_{2} \sin ^{4}(\theta)$, where we find that $K_{1}$ with $2.67 \mathrm{meV}$ is more than one order of magnitude larger than $K_{2}$ with $0.13 \mathrm{meV}$. Employing the LDA functional (for the same PBE-relaxed unit cell) yields a somewhat larger value of $3.11 \mathrm{meV} /$ f.u. (which is virtually unchanged if we calculate the MAE for the slightly smaller lattice parameters used in Ref. [28]), again in good agreement with recent calculations (reporting values from 2.85 to $3.12 \mathrm{meV} /$ f.u., depending on method and code) [28]. The local magnetic moments as well as the orbital moments calculated with PBE, LDA, and $\mathrm{LDA}+U$ can be found in Table I.

\section{Analyzing correlation effects}

Although we are able to reproduce the results of Khan et al. very well with less computational effort, we fail to reproduce the $\mathrm{LDA}+U$ results published previously by Shick and Myrasov [35]. Using their lattice parameters and values for both $U$ and $J$, we calculate a MAE of $2.79 \mathrm{meV} /$ f.u., which is about $0.32 \mathrm{meV}$ less than our result with LDA for their lattice parameters, but still about twice as large as their published result of $1.3 \mathrm{meV} /$ f.u.. Switching from the rotationally invariant LDA $+U$ flavor of Liechtenstein et al. [49], to the simplified version by Dudarev et al. [50], did not change our result significantly.

To further investigate the effects of correlation and possibly corroborate our $\mathrm{LDA}+U$ results, we employ the adiabatic connection fluctuation-dissipation theorem in the random phase approximation (ACFDT-RPA), as implemented in the VASP package [51-53]. Here, the correlation energy is computed via the plasmon fluctuation equation by calculating the independent particle response functions using occupied and unoccupied states. The exchange energy is calculated by Hartree-Fock theory. Both contributions are calculated nonself-consistently using DFT orbitals and are added to Hartree, kinetic, and Ewald energies to obtain the total ACFDT-RPA energy. Thus both local and orbital magnetic moments do not change with this method with respect to standard DFT, but the correlation and the exchange energies are calculated with significantly more evolved methods. The ACFDT-RPA is a nonlocal method that captures a significant portion of correlation effects (all ring-type Goldstone diagrams of manybody perturbation theory) and has been successfully used to improve on results of standard DFT without introducing external parameters $[54,55]$. As prudent for metallic systems, we neglected long wavelength contributions [53].

Due to the huge computational effort needed for such calculations, we where not able to perform them with the same accuracy as our other MAE calculations. ${ }^{2}$ Furthermore, the integral over $\omega$ in the plasmon equation has to be solved numerically using a fixed number of sampling points $N_{\omega}$. Fortunately, convergence with respect to $N_{\omega}$ (which can be troublesome for metals) was quick for FePt, and the necessary accuracy $(\sim 0.1 \mathrm{meV})$ was already obtained for $N_{\omega}=10$. Calculating the RPA energy for significantly more than $\sim 1000$ $k$ points proved impossible with our computational resources, so we reduced the $r_{\text {min }}$ parameter to maximally $34 \AA$, resulting in $1088 k$ points in the full Brillouin zone. The plane wave cutoff was also reduced to $600 \mathrm{eV}$, which then leads to an MAE of $3.1 \mathrm{meV} /$ f.u. on the PBE level, about $10 \%$ higher than for our converged computational parameters.

As can be seen from Fig. 2, the ACFDT-RPA calculations are not converged at $1088 k$ points, much in contrast to standard DFT, which is qualitatively correct even for comparatively low $k$-mesh densities and for an energy cutoff of $600 \mathrm{eV}$. For example, $420 k$ points are enough to approach the converged value of the MAE within $\sim 10 \%$ for PBE, while on the RPA level not even the easy axis is correctly predicted. However, the oscillations observed in the data above $600 k$ points allow us to estimate a region of convergence by fitting two linear functions to envelope the data (see grey area in Fig. 2). While this is not the most accurate estimate, we are fairly confident that the present data are sufficient to predict a higher value for the MAE than $2.0 \mathrm{meV} /$ f.u..

\footnotetext{
${ }^{2}$ The leftmost point (420 $k$ points) in Fig. 2 alone consumed over 12000 core hours, with the rightmost points exceeding this number by more than a factor of 10 !
} 


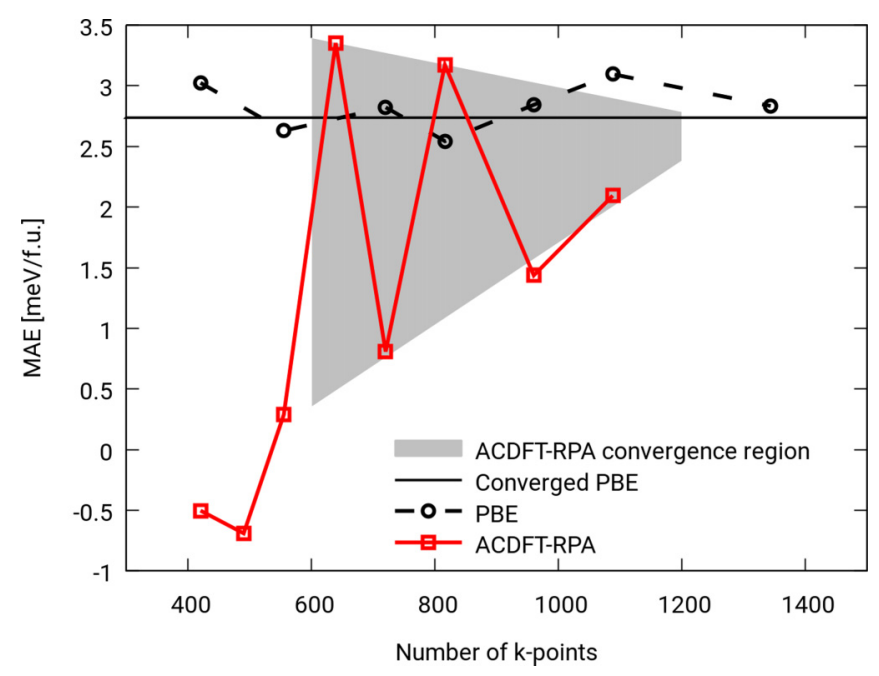

FIG. 2. MAE calculated on the ACFDT-RPA level with respect to the number of $k$ points in the full Brillouin zone. The PBE results for an energy cutoff of $600 \mathrm{eV}$ are also plotted for comparison. The MAE converges slowly with increased number of $k$ points but we estimate it to be higher than $2.0 \mathrm{meV} /$ f.u. (see grey region).

Although we could not fully converge our ACFDT-RPA calculations, the trend we observe, as well as our $\mathrm{LDA}+U$ results, make us confident that correlation effects alone are not able to reduce the MAE of FePt by a factor of two. In the following section, we will show that disorder is able to reconcile experiment and calculations much more satisfyingly than a high-level treatment of exchange and correlation.

\section{B. Defects in FePt}

As discussed in Sec. I, experimental measurements of the MAE of FePt are always performed for a somewhat disordered crystal. Disorder in a crystal can be quantified by the longrange order parameter $S$. In the case of a stoichiometric $\mathrm{FePt}$ crystal, the fractions of $\mathrm{Fe}$ and $\mathrm{Pt}$ atoms sitting on their correct respective lattice sites must be equal $\left(r_{\mathrm{Fe}}=r_{\mathrm{Pt}}=r\right)$, thus the equation for $S$ reduces to

$$
S=r_{\mathrm{Fe}}+r_{\mathrm{Pt}}-1=2 r-1 .
$$

For a totally disordered crystal $S=0$, as each atom has $50 \%$ probability to sit on its preferred lattice site, while $S=1$ is achieved for perfect order. ${ }^{3}$ Experimentally, the order parameter usually is estimated by the relative strength of integrated x-ray diffraction peaks $I_{001}$ and $I_{002}$ according to the formula

$$
S^{2}=\frac{\left(I_{001} / I_{002}\right)_{\text {meas }}}{\left(I_{001} / I_{002}\right)_{\text {calc }}^{S=1}},
$$

where the numerator consists of the measured values and the denominator uses calculated intensities for perfect order, assuming atomic scattering factors, Debye-Waller correction, Lorentz polarizations factors and structure factors [15]. However, in a recent investigation of a multigrain FePt nanoparticle

\footnotetext{
${ }^{3}$ Sometimes also the short-range order parameter $\eta$ is used in this context. $S$ and $\eta$ are connected by the relation $\eta=2 S-1$ and $0.5 \leqslant \eta \leqslant 1$.
}

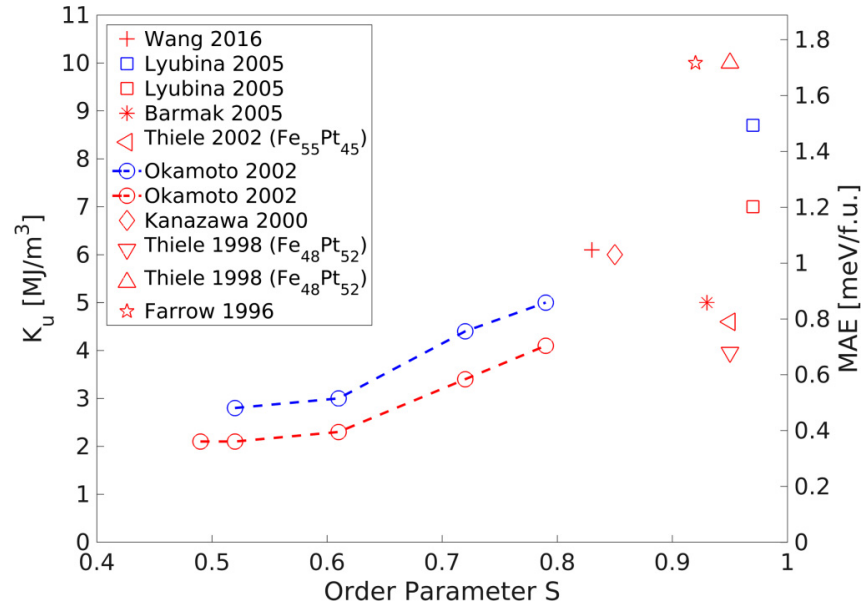

FIG. 3. Experimental anisotropy constant $K_{u}$ in $\mathrm{MJ} / \mathrm{m}^{3}$ and plotted over the long-range order parameter $S$. The right axis is a conversion to the MAE in meV. Data are taken from Refs. [11-17,31] as indicated in the legend. Red symbols are measurements at room temperature, while blue symbols stand for low temperature.

by $3 \mathrm{D}$ atomic electron tomography, it was observed that $\mathrm{L} 1_{0}$ order might be wrongly attributed in standard 2D methods due to overlapping $\mathrm{L}_{2}$ grains, although this seems unlikely in highly stoichiometric samples [56]. In Fig. 3, we have plotted several experimentally determined values for the magnetic anisotropy constant $K_{u}=K_{1}+K_{2}$. Values are given in $\mathrm{MJ} / \mathrm{m}^{3}$ and have also been converted to $\mathrm{meV} / \mathrm{f}$.u., for easier comparison to calculations. Most measurements have been performed at room temperature (RT shown with red symbols) but Okamoto et al. [12] and Lyubina et al. [31] have also provided low-temperature measurements at 10 and $5 \mathrm{~K}$, respectively (LT shown with blue symbols). From their data, we see that the MAE is reduced by $\sim 20 \%$ to $30 \%$ at RT compared to LT. More generally, the temperature dependence of the first-order anisotropy constant $K_{1}$ is coupled to the temperature dependence of the magnetization $M_{S}$ to approximately second power, $K_{1}(T) / K_{1}(0)=\left(M_{S}(T) / M_{S}(0)\right)^{2}$, as measured by Refs. [12,13] and calculated by Refs. [24,37,57,58]. From Fig. 3, we also see that the spread of values for high-order parameters is quite large, an effect which can be explained in part by the different compositions of the samples (see legend of Fig. 3), but also indicates the difficulties in accurately measuring such a large anisotropy with usual laboratory fields. For example, Thiele et al. [14] give two values for the MAE of the same sample, once measured by torque magnetometry (3.96 MJ $/ \mathrm{m}^{3}, \nabla$ in Fig. 3), and once deduced from saturation magnetization and dipolar length measurements $\left(10 \mathrm{MJ} / \mathrm{m}^{3}\right.$, $\triangle$ in Fig. 3), which differ by more than $100 \%$.

Computational studies investigating the MAE of disordered $\mathrm{FePt}$ in the $\mathrm{L} 1_{0}$ structure have been conducted by Staunton [59], Burkert [22], Kota [29], and their respective coworkers. ${ }^{4}$ Several studies also deal with the electronic structure and

\footnotetext{
${ }^{4}$ Reference [24] also provides data for disordered structures, but since they do not deviate from Ref. [59], other than for $S=1$, and two of the authors appear on both papers, we discuss only the earlier reference at this point.
} 


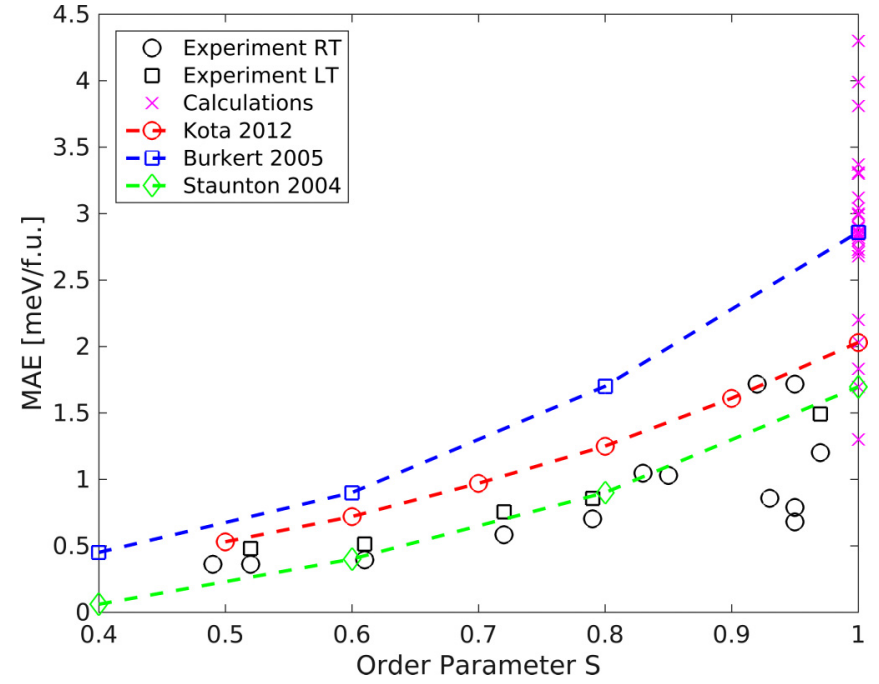

FIG. 4. MAE in $\mathrm{meV} /$ f.u. plotted over the long-range order parameter $S$. Purple crosses are the $a b$ initio results for the fully ordered system as presented also in Fig. 1. Black symbols represent experiments at room temperature (circles) and around $10 \mathrm{~K}$ (squares). The red circles, blue squares, and green diamonds represent the calculations by Kota [29], Burkert [22], and Staunton [59].

magnetic properties of the fully disordered alloy in the face centered cubic structure (e.g., Ref. [60] and the references therein). Generally, the coherent potential approximation (CPA) was used in these papers to model the disorder effects on a mean field basis. While the results calculated by Refs. [29,59] fit the experimental data very well (see Fig. 4), they arrive at considerably lower MAE values for the fully ordered system than the majority of other calculations, including the most recent study by Khan and coworkers [28]. Furthermore, at certain order parameters, some experimental data points lie higher than the CPA calculations, which seems unlikely given that surface effects, grain boundaries and varying grain orientations in experimental samples will likely decrease the MAE compared to the infinite crystal size of the calculations. Burkert et al. report data that agree with the most accurate calculations for full order and approach the experimental data nicely for lower values of $S$ (see Fig. 4). However, the mean-field approach is unable to predict which types of defects are responsible for the significant drop of $K_{u}$ with decreasing order and the divergence between the studies by Staunton, Kota, and Burkert, all using very similar methods, is a little unsatisfactory.

We, on the other hand, are more interested in the influence of single localized defects in the FePt crystal, especially antisite defects (ASD) and stacking faults (SF). An ASD consists of one $\mathrm{Fe}$ and one $\mathrm{Pt}$ atom exchanging their place in the lattice, while a SF can occur during growth of FePt thin films if instead of perfect alternating stacking of Fe and Pt planes, two planes of the same material follow each other. We distinguish between localized defects, where two neighboring atoms are exchanged for ASDs and two layers of one type are followed by two layers of the other in SF, and dispersed defects, where the exchanged atoms and the double-planes are far away from each other. These basic defects are depicted in Fig. 5.

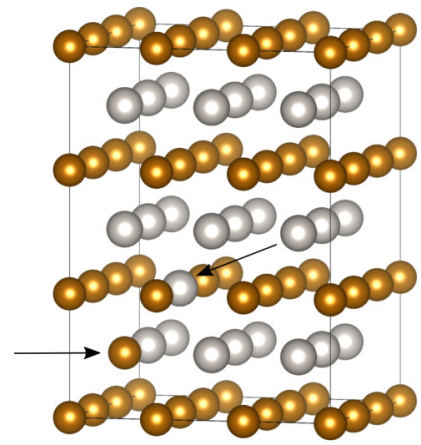

(a)

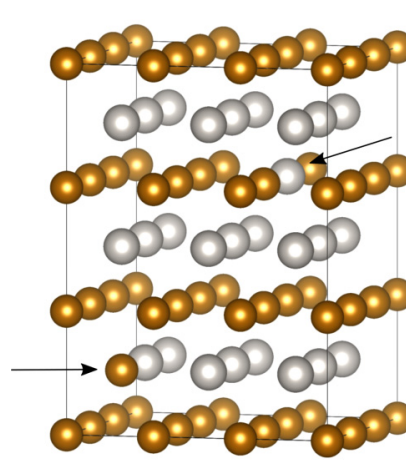

(b)

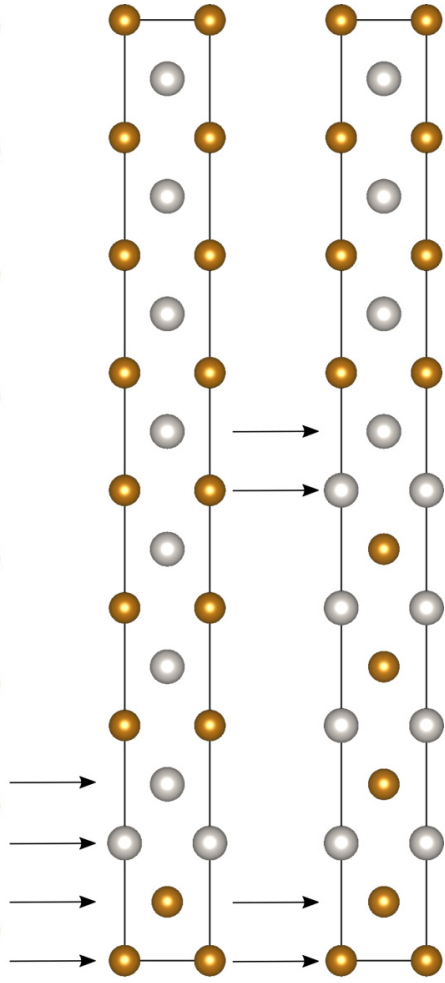

(c)

FIG. 5. Depiction of a localized (a) and a dispersed (b) antisite defect, as well as a localized (c) and a dispersed (d) stacking fault in stoichiometric FePt alloy. ASDs are modeled in a 54 atom and SFs in a 16 atom supercell, with Fe shown in gold and Pt in silver. Arrows mark the defect positions.

\section{Defect formation energies}

While we do not consider a change in cell volume or cell shape, the atomic positions in all of our supercells have been relaxed carefully and separate static calculations are used to determine the defect formation energies. As we only consider defects where two (or more) atoms exchange their positions and keep the alloy fully stoichiometric, the defect formation energy (DFE) is simply the total energy of the supercell containing the defect minus $n$ times the total energy of a fully relaxed FePt unit cell, where $n$ is the number of FePt pairs in the supercell, $E_{\mathrm{df}}=E_{\mathrm{sc}}-n E_{\mathrm{uc}}$. Keeping the supercell fixed simplifies the treatment of symmetry breaking by defects, which only becomes important if more than one defect is considered per unit cell. Thus we avoid complex ensemble averages that have to be considered if random disorder is modeled with a supercell approach [61]. Defect formation energies for different super cell sizes (described as multiples of the unit cell in $a, b$, and $c$ direction) are given in Table II for both ASDs and SFs. If two defects are considered in a cell the DFEs are averaged over several configurations. For example, if two local ASDs are put into a $2 \times 2 \times 2$ supercell with the first one being located at the origin, the second on can be shifted by a lattice vector $a$ (equivalent to a shift by $b$ ), a lattice vector $c$, by both $a$ and $c$ (equivalent to $b$ and $c$ ), $a$ and $b$, or by $a$, $b$, and $c$ together. We thus arrive at five different possibilities, of which two have to be counted twice since they have less 
TABLE II. Defect formation energies $E_{\mathrm{df}}$ per defect for ASDs and SFs with corresponding order parameters $S$ in different supercells (SC) and configurations. $N$ is equal to the number of defects per cell, while $\mathrm{L}$ or $\mathrm{D}$ denote a local or a dispersed defect.

\begin{tabular}{ccccc}
\hline \hline \multicolumn{5}{c}{ ASDs } \\
SC & $N$ & L/D & $S$ & $E_{\mathrm{df}} / N(\mathrm{meV})$ \\
\hline $4 \times 4 \times 4$ & 1 & $\mathrm{~L}$ & 0.97 & 735.6 \\
$3 \times 3 \times 3$ & 1 & $\mathrm{~L}$ & 0.93 & 781.1 \\
$3 \times 3 \times 3$ & 1 & $\mathrm{D}$ & 0.93 & 938.5 \\
$3 \times 3 \times 3$ & 2 & $\mathrm{~L}$ & 0.85 & 714.6 \\
$2 \times 2 \times 2$ & 1 & $\mathrm{~L}$ & 0.75 & 745.5 \\
$2 \times 2 \times 2$ & 2 & $\mathrm{~L}$ & 0.50 & 566.9 \\
& & & & \\
$\mathrm{SC}$ & $N$ & $\mathrm{~L} / \mathrm{D}$ & $S$ & $E_{\mathrm{df}} / N(\mathrm{meV})$ \\
$1 \times 1 \times 12$ & 1 & $\mathrm{~L}$ & 0.83 & 449.4 \\
$1 \times 1 \times 10$ & 1 & $\mathrm{~L}$ & 0.80 & 451.6 \\
$1 \times 1 \times 10$ & 1 & $\mathrm{D}$ & 0.80 & 455.8 \\
$1 \times 1 \times 8$ & 1 & $\mathrm{~L}$ & 0.75 & 451.6 \\
$1 \times 1 \times 8$ & 1 & $\mathrm{D}$ & 0.75 & 453.5 \\
$1 \times 1 \times 6$ & 1 & $\mathrm{~L}$ & 0.66 & 449.4 \\
$1 \times 1 \times 5$ & 1 & $\mathrm{~L}$ & 0.60 & 450.2 \\
$1 \times 1 \times 4$ & 1 & $\mathrm{~L}$ & 0.50 & 453.9 \\
$1 \times 1 \times 3$ & 1 & $\mathrm{~L}$ & 0.33 & 447.1 \\
$1 \times 1 \times 2$ & 1 & $\mathrm{~L}$ & 0.00 & 443.2 \\
\hline \hline
\end{tabular}

symmetry. The DFEs are actually quite different, ranging from $435 \mathrm{meV}$ for stacking along $c$, to $690 \mathrm{meV}$ for stacking along $a$ or $b$.

We immediately notice that SFs have a lower DFE than ASDs, and that they are very well decoupled from each other, since the energy stays nearly constant at $\sim 450 \mathrm{meV}$. If the stacking fault is localized, with two Pt layers followed immediately by two Fe layers, or dispersed, where the double layers are far away from each other does not matter much from an energetic point of view. On the other hand, a single local ASD shows quite different $E_{\mathrm{df}}$ depending on supercell size. For the $3 \times 3 \times 3$ supercell, the DFE is noticeable higher than for the largest cell considered, but the $2 \times 2 \times 2$ shows again a reduced $E_{\mathrm{df}}$. This indicates that ASDs are not decoupled, and interact attractively in close proximity, as can be seen from the averaged DFE for two ASDs in a $2 \times 2 \times 2$ supercell, which, at $\sim 566 \mathrm{meV}$ per defect, is considerably lower than an isolated ASD $(\sim 736 \mathrm{meV})$.

In a very recent study, Yang et al. used high-resolution, tomographic, scanning transmission electron microscope data to map the positions of over $23000 \mathrm{Fe}$ and $\mathrm{Pt}$ atoms in a multigrain FePt nanoparticle [56]. ASDs are frequently observed in their non stoichiometric sample, while SFs are not seen at all. ${ }^{5}$ This seems to contradict our data for DFEs, which predict SF to occur more likely than SFs. However, one has to keep in mind that a SF is an extended defect, ranging over the whole grain (or in our model over the whole infinite crystal).

\footnotetext{
${ }^{5}$ In the nonstoichiometric sample of Ref. [56], a swap defect denotes what we call here ASD. An antisite defect in their notation is just a single Fe atom sitting on a Pt site or vice versa. Here we call this type of defect "point defect."
}

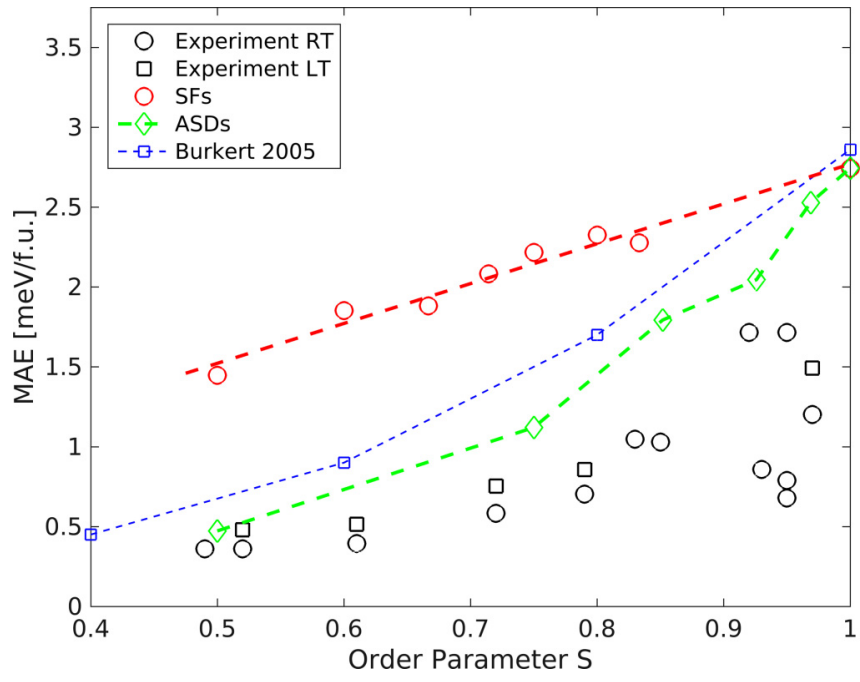

FIG. 6. MAE in meV/f.u. plotted over the long-range order parameter $S$. Black symbols represent experiments at room temperature (circles) and around $10 \mathrm{~K}$ (squares). The red circles are for SFs, green diamonds are for ASDs, and blue squares are the KKR-CPA results of Ref. [22]. SF data are fitted with a straight line, all other lines serve only as guides to the eye.

Thus the likelihood of SFs forming during the transition from the disordered $A 1$ phase to the $L 1_{0}$ phase is significantly lower than the survival of local ASDs. Furthermore, Ref. [56] report a large number of point defects, which is a single $\mathrm{Fe}(\mathrm{Pt})$ atom sitting on a $\mathrm{Pt}(\mathrm{Fe})$ lattice site. This differs from an ASD, where one $\mathrm{Fe}$ and one $\mathrm{Pt}$ atom exchange places, as it breaks stoichiometry. Estimating the DFE of a point defect as half of the DFE of a dispersed ASD, we arrive at $\sim 470 \mathrm{meV}$, which is comparable to the DFE of SFs.

From the data of Table II, we see that the DFEs are quite sizable at $\sim 0.5$ to $0.9 \mathrm{eV}$ per defect, depending on type and configuration. Thus healing out defects in a FePt alloy with low-order parameter $S$ would lower the free energy enormously, even considering the decreased entropy. The difficulties in producing highly ordered films of the material experimentally (see Sec. I), leads thus to the conclusion that the barriers for defect healing must be comparably large.

\section{Magnetic anisotropy energy}

The magnetic anisotropy energy was calculated for the supercells of Table II analogous to the method used for the perfect crystal with the same energy cutoff and $k$-grid density. Our results for both SFs and ASDs are plotted in Fig. 6 alongside the mean-field data from Burkert et al. [22] and the experimental data detailed in Fig. 3.

It is obvious that although the DFE of ASDs is higher than for SFs, the former are responsible for the strong decrease of the MAE at reduced order. For stacking faults, a decrease in $S$ leads to a linear reduction of the MAE. This means that the defects are not only well isolated from each other regarding the DFE, but also regarding the MAE. As the fraction of correctly ordered unit cells in the supercell decreases, the MAE decreases proportionally, right down $S=0.5$. If the cell size is further reduced to six atoms ( $S=0.33$ ) or four atoms $(S=0)$, 
which are not shown in Fig. 6, it is not really appropriate to speak of a SF, as $2 / 3$ or more of the material is layered in the wrong way. For $S=0.33$, we calculate an MAE of $1.40 \mathrm{meV}$, slightly higher than the linear trend would predict, while for $S=0$ the MAE drops to $0.18 \mathrm{meV}$. An antisite defect on the other hand, has much larger effect, which can also be reasoned intuitively, as there are significantly more unit cells directly influenced by a single localized ASD (8) than by a SF (2), disregarding lower-order effects like atoms sitting on wrong lattice sites in a neighboring unit cell or relaxations. Furthermore, ASDs perturb the surrounding of $\mathrm{Pt}$ atoms (which are mainly responsible for the large MAE) in $3 \mathrm{D}$, while SFs only change the surrounding in 2D, having a diminished effect on the MAE. While our calculations for ASDs are in reasonable agreement with the experimental data over the whole range, the agreement is certainly a lot better for lower values of $S$. This might indicate that ASDs do indeed cluster together in $\mathrm{FePt}$, as our data for $S=0.5$ are averaged over different configurations of two ASDs in an 16 atom supercell. This result is also supported by the DFE data in Table II.

The KKR-CPA results from Burkert et al. [22] lies between our SF and ASD data, although closer to the ASD points. This is to be expected from a mean field approach for random disorder, which should encounter SF like regions less than ASDs. Delocalized defects where not included in Fig. 6, due to generally higher DFE, but the cases that we tested showed MAE differing less than 5\% from the localized defects in the same supercell.

In the single-atom resolution images from Ref. [56], ASDs are also commonly observed and their density is still $\sim 3 \%$ in the highest ordered grain centers of the nanoparticle. This is a strong indication that ASDs are also common in stoichiometric FePt, although they have a rather large DFE. Although we can not completely rule out that the MAE might also be lowered slightly by correlation effects (see Sec. III A 1), we believe that the inclusion of ASDs is sufficient to explain the experimental MAE data on the basis of standard DFT calculations.

\section{CONCLUSIONS}

We have shown that ASDs are responsible for the large reduction of the MAE in FePt with decreasing long-range order parameter $S$. Experimental measurements and $a b$ initio DFT calculations are thus also comparable without including many body effects beyond the LDA or GGA level. Qualitative calculations using the ACDFT-RPA show that the effect of more accurate treatment of correlations is probably smaller than that of disorder. This will allow future DFT calculations to accurately model thin FePt films and layered systems useful for magnetic recording with reasonable effort.

\section{ACKNOWLEDGMENTS}

The authors would like to thank J. Redinger and H. Michor for fruitful discussions, and acknowledge the support by the Austrian Science Fund (FWF) [SFB ViCoM F4109-N28 and F4112-N28]. The ample support of computer resources by the Vienna Scientific Cluster (VSC) are gratefully acknowledged. Figure 5 in this paper was created with help of the VESTA code [62].
[1] Y. Ding, X. Zhao, and E. Liu, Magnetic Thin Films: Properties, Performance and Applications (Nova Science, New York, 2011), Chap. 8.

[2] D. Weller, O. Mosendz, G. Parker, S. Pisana, and T. S. Santos, Phys. Status Solidi A 210, 1245 (2013).

[3] D. Weller, G. Parker, O. Mosendz, E. Champion, B. Stipe, X. Wang, T. Klemmer, G. Ju, and A. Ajan, IEEE Trans. Magn. 50, 1 (2014).

[4] H. J. Richter, A. Lyberatos, U. Nowak, R. F. L. Evans, and R. W. Chantrell, J. Appl. Phys. 111, 033909 (2012).

[5] M. Kryder, E. Gage, T. McDaniel, W. Challener, R. Rottmayer, G. Ju, Y.-T. Hsia, and M. Erden, Proc. IEEE 96, 1810 (2008).

[6] D. Suess, T. Schrefl, S. Fähler, M. Kirschner, G. Hrkac, F. Dorfbauer, and J. Fidler, Appl. Phys. Lett. 87, 012504 (2005).

[7] D. Suess, Appl. Phys. Lett. 89, 113105 (2006).

[8] M. Wolloch, M. E. Gruner, W. Keune, P. Mohn, J. Redinger, F. Hofer, D. Suess, R. Podloucky, J. Landers, S. Salamon, F. Scheibel, D. Spoddig, R. Witte, B. Roldan Cuenya, O. Gutfleisch, M. Y. Hu, J. Zhao, T. Toellner, E. E. Alp, M. Siewert, P. Entel, R. Pentcheva, and H. Wende, Phys. Rev. B 94, 174435 (2016).

[9] J.-U. Thiele, S. Maat, J. Robertson, and E. E. Fullerton, IEEE Trans. Magn. 40, 2537 (2004).

[10] G. Ju, Y. Peng, E. K. Chang, Y. Ding, A. Q. Wu, X. Zhu, Y. Kubota, T. J. Klemmer, H. Amini, L. Gao et al., IEEE Trans. Magn. 51, 1 (2015).
[11] K. Barmak, J. Kim, L. H. Lewis, K. R. Coffey, M. F. Toney, A. J. Kellock, and J.-U. Thiele, J. Appl. Phys. 98, 033904 (2005).

[12] S. Okamoto, N. Kikuchi, O. Kitakami, T. Miyazaki, Y. Shimada, and K. Fukamichi, Phys. Rev. B 66, 024413 (2002).

[13] J.-U. Thiele, K. R. Coffey, M. F. Toney, J. A. Hedstrom, and A. J. Kellock, J. Appl. Phys. 91, 6595 (2002).

[14] J.-U. Thiele, L. Folks, M. F. Toney, and D. K. Weller, J. Appl. Phys. 84, 5686 (1998)

[15] J. Wang, H. Sepehri-Amin, Y. Takahashi, S. Okamoto, S. Kasai, J. Kim, T. Schrefl, and K. Hono, Acta Mater. 111, 47 (2016).

[16] R. F. C. Farrow, D. Weller, R. F. Marks, M. F. Toney, A Cebollada, and G. R. Harp, J. Appl. Phys. 79, 5967 (1996).

[17] H. Kanazawa, G. Lauhoff, and T. Suzuki, J. Appl. Phys. 87, 6143 (2000).

[18] T. Shiroyama, J. Wang, A. Felicia, Y. Takahashi, and K. Hono, J. Magn. Magn. Mater. 432, 129 (2017).

[19] C. J. Aas, L. Szunyogh, J. S. Chen, and R. W. Chantrell, Appl. Phys. Lett. 99, 132501 (2011).

[20] C. J. Aas, P. J. Hasnip, R. Cuadrado, E. M. Plotnikova, L. Szunyogh, L. Udvardi, and R. W. Chantrell, Phys. Rev. B 88, 174409 (2013).

[21] C. J. Aas, L. Szunyogh, and R. W. Chantrell, Europhys. Lett. 102, 57004 (2013)

[22] T. Burkert, O. Eriksson, S. I. Simak, A. V. Ruban, B. Sanyal, L. Nordström, and J. M. Wills, Phys. Rev. B 71, 134411 (2005). 
[23] G. H. O. Daalderop, P. J. Kelly, and M. F. H. Schuurmans, Phys. Rev. B 44, 12054 (1991).

[24] A. Deák, E. Simon, L. Balogh, L. Szunyogh, M. dos Santos Dias, and J. B. Staunton, Phys. Rev. B 89, 224401 (2014).

[25] I. Galanakis, M. Alouani, and H. Dreyssé, Phys. Rev. B 62, 6475 (2000).

[26] M. E. Gruner, Phys. Status Solidi A 210, 1282 (2013).

[27] A. Kabir, J. Hu, V. Turkowski, R. Wu, R. Camley, and T. S. Rahman, Phys. Rev. B 92, 054424 (2015).

[28] S. Ayaz Khan, P. Blaha, H. Ebert, J. Minár, and O. Šipr, Phys. Rev. B 94, 144436 (2016).

[29] Y. Kota and A. Sakuma, J. Phys. Soc. Jpn. 81, 084705 (2012).

[30] H. B. Luo, W. X. Xia, A. V. Ruban, J. Du, J. Zhang, J. P. Liu, and A. Yan, J. Phys.: Condens. Matter 26, 386002 (2014).

[31] J. Lyubina, I. Opahle, K.-H. Müller, O. Gutfleisch, M. Richter, M. Wolf, and L. Schultz, J. Phys.: Condens. Matter 17, 4157 (2005).

[32] S. Ostanin, S. S. A. Razee, J. B. Staunton, B. Ginatempo, and E. Bruno, J. Appl. Phys. 93, 453 (2003).

[33] P. Ravindran, A. Kjekshus, H. Fjellvåg, P. James, L. Nordström, B. Johansson, and O. Eriksson, Phys. Rev. B 63, 144409 (2001).

[34] A. Sakuma, J. Phys. Soc. Jpn. 63, 3053 (1994).

[35] A. B. Shick and O. N. Mryasov, Phys. Rev. B 67, 172407 (2003).

[36] I. V. Solovyev, P. H. Dederichs, and I. Mertig, Phys. Rev. B 52, 13419 (1995).

[37] J. B. Staunton, S. Ostanin, S. S. A. Razee, B. L. Gyorffy, L. Szunyogh, B. Ginatempo, and E. Bruno, Phys. Rev. Lett. 93, 257204 (2004).

[38] O. A. Ivanov, L. V. Solina, V. A. Demshina, and L. M. Magat, Fiz. Metal. Metalloved. 35, 92 (1973).

[39] G. Kresse and J. Hafner, Phys. Rev. B 47, 558 (1993).

[40] G. Kresse and J. Hafner, Phys. Rev. B 49, 14251 (1994).

[41] G. Kresse and J. Furthmüller, Comput. Mater. Sci. 6, 15 (1996).

[42] G. Kresse and J. Furthmüller, Phys. Rev. B 54, 11169 (1996).

[43] P. E. Blöchl, Phys. Rev. B 50, 17953 (1994).
[44] G. Kresse and D. Joubert, Phys. Rev. B 59, 1758 (1999).

[45] P. Wisesa, K. A. McGill, and T. Mueller, Phys. Rev. B 93, 155109 (2016).

[46] J. P. Perdew, K. Burke, and M. Ernzerhof, Phys. Rev. Lett. 77, 3865 (1996).

[47] M. Methfessel and A. T. Paxton, Phys. Rev. B 40, 3616 (1989).

[48] P. E. Blöchl, O. Jepsen, and O. K. Andersen, Phys. Rev. B 49, 16223 (1994).

[49] A. I. Liechtenstein, V. I. Anisimov, and J. Zaanen, Phys. Rev. B 52, R5467 (1995).

[50] S. L. Dudarev, G. A. Botton, S. Y. Savrasov, C. J. Humphreys, and A. P. Sutton, Phys. Rev. B 57, 1505 (1998).

[51] J. Harl and G. Kresse, Phys. Rev. B 77, 045136 (2008).

[52] J. Harl and G. Kresse, Phys. Rev. Lett. 103, 056401 (2009).

[53] J. Harl, L. Schimka, and G. Kresse, Phys. Rev. B 81, 115126 (2010).

[54] H. Eshuis, J. E. Bates, and F. Furche, Theor. Chem. Acc. 131, 1084 (2012).

[55] X. Ren, P. Rinke, C. Joas, and M. Scheffler, J. Mater. Sci. 47, 7447 (2012).

[56] Y. Yang, C.-C. Chen, M. C. Scott, C. Ophus, R. Xu, A. Pryor, L. Wu, F. Sun, W. Theis, J. Zhou, M. Eisenbach, P. R. C. Kent, R. F. Sabirianov, H. Zeng, P. Ercius, and J. Miao, Nature 542, 75 (2017)

[57] O. N. Mryasov, U. Nowak, K. Y. Guslienko, and R. W. Chantrell, Europhys. Lett. 69, 805 (2005).

[58] N. Kobayashi, K. Hyodo, and A. Sakuma, Jpn. J. Appl. Phys. 55, 100306 (2016)

[59] J. B. Staunton, S. Ostanin, S. S. A. Razee, B. Gyorffy, L. Szunyogh, B. Ginatempo, and E. Bruno, J. Phys.: Condens. Matter 16, S5623 (2004).

[60] S. A. Khan, J. Minár, H. Ebert, P. Blaha, and O. Šipr, Phys. Rev. B 95, 014408 (2017).

[61] J. Buschbeck, I. Opahle, S. Fähler, L. Schultz, and M. Richter, Phys. Rev. B 77, 174421 (2008).

[62] K. Momma and F. Izumi, J. Appl. Crystallogr. 44, 1272 (2011). 